\title{
EL GÉNERO COMO RECURSO POLÍTICO: EL USO ESTRATÉGICO DEL GÉNERO POR MICHELLE BACHELET (CHILE) Y LAURETTE ONKELINX (BÉLGICA)'
}

\author{
Bérengère Marques-Pereira ${ }^{2}$ \\ David Paternotte ${ }^{3}$ \\ Mariana Valenzuela ${ }^{4}$
}

\section{Resumen/Abstract}

Este artículo se enfoca en la utilización estratégica del género que desarrollan dos prominentes mujeres políticas: Michelle Bachelet en Chile y Laurette Onkelinx en Bélgica. A diferencia de la mayoría de los estudios del área de mujeres y política, el artículo destaca que el género no debe ser entendido solo como una barrera para su inclusión sino que debe ser visto como un recurso político en la medida en que las mujeres en política lo utilizan para perfilar de manera significativa su imagen pública. Bachelet y Onkelinx han intentado dotar de nuevos significados a la maternidad al proponer nuevas combinaciones de sus identidades como mujeres, esposas y madres, definiendo esos procesos en particular en función de los contextos socio-políticos: Bachelet necesitaba probar que ella podía ser una mujer en política sin dañar a la política, resignificando la femineidad como un aporte para ella, mientras que Onkelinx quería demostrar que ella podía ser una mujer en política sin dañar a la familia.

Palabras claves: género, recurso político, maternidad, Bachelet, Onkelinx.

\footnotetext{
1 El artículo es producto del proyecto "El desarrollo de la participación política de la mujer: el caso de Chile en América Latina y el caso de Bélgica y Francia (elementos comparativos con Alemania y Finlandia) en Europa", desarrollado entre la Universidad Libre de Bruselas (Bélgica) y la Universidad Academia de Humanismo Cristiano, entre los años 2007 y 2009.

2 Belga, Universidad Libre de Bruselas. Email: bmarques@ulb.ac.be

3 Belga, Universidad Libre de Bruselas. Email: david.paternotte@ulb.ac.be

4 Chilena, Universidad de Santiago. Email: mariana.valenzuela@usach.cl
} 
GENDER AS POLITICAL APPEAL: THE STRATEGIC USE OF GENDER MICHELLE BACHELET (CHILE) AND LAURETTE ONKELINX (BELGIUM)

This paper focuses on the strategic use of "gender" made by two salient female politic figures: Michelle Bachelet in Chile and Laurette Onkelinx in Belgium. It underscores that gender is not to be understood only as a barrier to inclusion but that it has become a salient political resource, as it is shown by the use made by women in politics when profiling their public images. However, this strategic use is defined regarding each particular socio-political context. Bachelet and Onkelinx have intended to give new meanings to maternity by proposing new combinations of their identities as women, wives and mothers. But, while Bachelet needed to prove that women in politics do not entail a threat to politics, Onkelix underscored the fact that being a woman and a politician does not entail a threat to family.

Keywords: gender, political resource, motherhood, Bachelet, Onkelinx.

En las últimas décadas la representación política de las mujeres ha sido ampliamente estudiada. Han sido investigados temas como las causas de la exclusión política de las mujeres (Pateman, 1998; Phillips, 1995; Young, 2000), la representación social de las mujeres en la política (Huddy \& Terkildsen, 1993), y las soluciones potenciales como las cuotas y las leyes de paridad (entre otros Archenti y Tula, 2008; Celis, Krook \& Meier, 2011; Krook, 2010; Lovenduski, 2005). También ha sido abordada su eficiencia y los diseños más favorables institucionales y políticos (Mateo Díaz, 2005), siendo en este contexto el poder legislativo una de las preocupaciones básicas (Tremblay, 2008; Marques-Pereira \& Nolasco 2001). Si bien, los estudios sobre mujeres en los ministerios y los cuerpos ejecutivos han sido escasos, ha habido un reciente boom en la investigación tanto a nivel global (ver Borelli, 2002; Clift \& Brazaitis, 2003; Coulomb-Gully, 2009a; Jalalzai, 2008; Jalalzai \& Krook, 2010; Murray, 2010; Sineau, 2011; Bauer \& Tremblay, 2011) como en América Latina (Doran, 2010; EscobarLemmon \& Taylor-Robinson, 2005, 2009; Marques-Pereira, 2007a, 2011). Es en esta última línea que se inscribe este texto. 
En este artículo nos enfocamos en dos prominentes mujeres políticas: Michelle Bachelet, quien fue dos veces ministra antes de ser elegida presidenta de Chile en 2006, y Laurette Onkelinx, quien es una de las vice-presidentas del gobierno belga desde 1999. Nos interesa identificar las formas en que ambas movilizan el género como un recurso político y la manera en que esta movilización se especifica en función de los contextos socio-políticos particulares.

El material presentado aquí proviene de un estudio sobre mujeres en los gobiernos de Europa y América Latina, abocado a identificar los discursos de género y formas de presentación en revistas y periódicos de circulación nacional. Lo que se presenta aquí son dos casos de mujeres socialistas que ocuparon puestos destacados en el ejecutivo de sus países en contextos regionales diferentes, en los que, a pesar de las diferencias y particularidades propias a cada cual, se ejemplifica el uso del género como recurso político. Los más importantes periódicos chilenos ${ }^{5}$ y belgas ${ }^{6}$ fueron analizados para el período en los cuales Bachelet y Onkelinx ejercieron sus puestos. En el caso belga nos centramos en el período en que Onkelinx era miembro del gobierno federal (desde 1999), con algunas referencias a momentos claves anteriores. En el caso chileno hemos examinado particularmente el período de la primera campańa electoral (2005) y primer gobierno de Bachelet (2006-2010), pero haciendo referencia a documentos previos a este momento en cuanto pertinentes a la argumentación. Adicionalmente, se rastrearon entrevistas dadas por ellas a revistas de circulación masiva. En ambos casos, las entrevistas aparecieron como la mejor manera de estudiar el género como recurso político pues el carácter dialógico de este género lo constituye como un espacio de mayor injerencia posible de las propias políticas en los modos de representación final. Finalmente, el artículo se nutre del debate académico principalmente en torno a la figura de Bachelet. La presidenta de Chile, quien fue la primera mujer en ejercer este cargo y la primera en llegar a la presidencia de un país latinoamericano sin tener

5 Se hizo una revisión de los cinco periódicos de mayor circulación del país: El Mercurio, La Cuarta, La Tercera, La Nación y Las Últimas Noticias, entre comienzos del 2006 y comienzos del 2008, seleccionándose los artículos y notas que se referían a la mandataria y las entrevistas que se publicaron de ella. También se hizo una búsqueda de entrevistas dadas por ella a diferentes medios impresos nacionales durante su época de ministra y candidata presidencial en las que hiciera referencia explícita al hecho de ser mujer, además de algunas entrevistas destacadas que dio hacia el final de su mandato.

6 Se llevó a cabo una revisión sistematica del mayor periódico en Bélgica francófona, Le Soir, y de los dossiers personales del Instituto Emile Vandervelde, vinculado al Partido Socialista, durante el período 1999-2009. Estos dossiers compilan todos los artículos y entrevistas referidos a la ministra publicados en Bélgica, tanto francófona como flamenca, así como prensa extranjera. 
lazos consanguíneos con políticos masculinos (Llanos y Sample, 2008), ha llamado la atención en la comunidad académica global (Franceschet, 2006; Franceschet \& Thomas, 2010; Vera Guajardo, 2008; Thomas, 2011; Thomas \& Adams, 2010; Valenzuela y Correa, 2009). Al contrario, la figura de Onkelinx no ha sido abordada desde una perspectiva de género.

$\mathrm{Al}$ afrontar las maneras en que Bachelet y Onkelinx trataron de perfilar su imagen en los medios, este artículo intenta sumarse a investigaciones que buscan superar una tendencia en buena parte de los estudios sobre mujeres y política, que abordaban, principalmente, los obstáculos que encontraban las mujeres en la política. Si bien esta entrada ha sido extremadamente útil, este tipo de investigación ha perpetuado con frecuencia la imagen de mujeres como víctimas, aún en los estudios de mujeres en el gobierno (Hardy, 2005; Sineau, 2011). Por ejemplo, en su libro pionero que examinaba las representaciones sociales sobre Édith Cresson -la primera mujer que fue Primer Ministro en Francia (1991-1992)-, y Margaret Thatcher -la primera mujer que ha sido Primer Ministro en Gran Bretaña (1979-1990)-, Jane Freedman (1997) consideraba que la primera fue representada como exponente de todas las fallas generalmente atribuidas a las mujeres mientras que la segunda se distanció de todas las cualidades femeninas que fueron pensadas en cierto momento como incompatibles con el éxito político (p. 229). En ambos casos, las mujeres eran vistas más como víctimas que como protagonistas, permitiéndoles las representaciones asociadas a las mujeres en el poder, en el mejor de los casos, un poder de influencia pero no de autonomía.

No negamos que las mujeres todavía encaran barreras estructurales cuando tratan de entrar o de avanzar en la política. Sin embargo, la situación ha cambiado y enfocarse demasiado en estas barreras nos llevaría a no ver una parte significativa del asunto. Primero, los términos del debate político se han alterado radicalmente y el tema de la participación política de las mujeres es discutido globalmente. Segundo, las mujeres participan crecientemente en política y algunos países incluso han adoptado cuotas para aumentar su presencia. En tercer lugar, como se muestra en los casos de Bachelet y Onkelinx, a pesar que no sean numerosas, las mujeres tienen acceso a las más altas esferas del poder. En consecuencia, no se puede estudiar el género en la política como se hacía hace algunos años. Las mujeres en política no pueden ser consideradas solamente víctimas del patriarcado y del sexismo sino también deben ser vistas como actoras relevantes de la actividad política. En este nuevo contexto necesitamos investigar de qué manera estas destacadas mujeres políticas se presentan en las arenas políticas 
sociales y cómo se relacionan con el género. El género en este contexto no es solamente una barrera sino que debe ser visto como un recurso político en la medida en que las mujeres en política lo utilizan para perfilar de manera significativa su imagen pública aun cuando ellas no hayan sido víctimas de ataques sexistas. De hecho, como lo demuestran los casos estudiados, tanto Bachelet como Onkelinx efectuaron una utilización estratégica del género para promover su carrera política.

\section{El género como recurso y los contextos diferenciales}

Nuestra tesis hace eco de estudios desarrollados en la última década. Algunos investigadores e investigadoras han subrayado que el género es con frecuencia un área de interés de las mujeres políticas que habría sido utilizado o reconfigurado de manera ventajosa (Bonnafous, 2003; Collomb-Gully, 2009b; Thomas \& Adams, 2010, entre otros). Por ejemplo, en su investigación acerca de las candidatas mujeres durante las elecciones municipales de 2001 en París, Delphine Dulong y Sandrine Lévêque (2002) examinaron los efectos de la ley de paridad y mostraron que el género puede ser utilizado como un recurso estratégico para la representación de sí durante las campañas electorales. Más recientemente algunos estudios de caso enfocados en políticas mujeres líderes han subrayado de qué forma han intentado influir en las maneras en que su género ha sido percibido. Por ejemplo, en un estudio sobre la construcción del vestido tomado como una metáfora del género en la carrera de Margaret Thatcher, Daniel Conway (2011) argumenta que el foco puesto en la vestimenta era potencialmente pero no inevitablemente un factor de daño o marginalización. La entonces Primer Ministro demostró diferentes niveles de agencia sobre su vestimenta y el rol que ésta tenía en su presentación/representación como mujer en política. Estas aproximaciones no solamente se acercan al género como un régimen político, un conjunto de obstáculos y una matriz de inteligibilidad que sería impuesta a los individuos y que los trascendería sino que consideran también que el género es encarnado y performado por los individuos dando espacio para el cambio y la agencia.

Obviamente este uso estratégico del género se inscribe en un marco común latinoamericano y europeo, chileno y belga. Este marco proviene de las consecuencias de las conferencias de Beijing y la ratificación de la CEDAW que reconocen la igualdad entre hombres y mujeres. Este logro fue posible por un proceso histórico de construcción del sujeto político femenino basado en actividades controversiales y conflictivas. Estas dinámicas han 
politizado lo privado y lo doméstico al cuestionar la naturalización de los roles y las funciones sociales o la aparente evidencia de la separación de roles al mismo tiempo de hacer visibles las relaciones de género y su dialéctica de exclusión/inclusión. Este proceso colectivo se basó en la construcción de una autonomía afirmada en la emancipación de todas formas de tutelaje paternal o marital. Una emancipación que libera a cada mujer y la deja libre para relacionarse o involucrarse en grupos sociales y políticos o instituciones para entrar en nuevas formas de relaciones de poder (Marques Pereira, 2003). De esta manera, la capacidad de las mujeres para manejar las relaciones de poder en las esferas públicas y políticas comenzó a ser concebible e incluso posible. En los noventa, la casi ausencia de mujeres en política, con la excepción de los países nórdicos, dejó de ser un tema silenciado y comenzó ha convertirse en un verdadero asunto político. La Conferencia de Beijing trajo este asunto de la representación política de las mujeres a la primera plana. Quince años después, la presencia y la voz de las mujeres en los cuerpos ejecutivos, incluyendo los más altos niveles del Estado, se ha convertido por lo tanto en un tema público. La puesta en cuestión del monopolio del poder masculino en política se refleja no solamente en una creciente presencia de las mujeres en funciones ejecutivas sino también en el hecho que los actores femeninos políticos no son outsiders de la política. Ellas han sido capaces de construir su propio capital político y de tornar el género en un recurso estratégico. En particular, tal como va a ser discutido aquí, han intentado re-significar la maternidad al proponer nuevas combinaciones de sus identidades como mujeres, esposas y madres. Sin embargo, estos procesos se definen de manera particular en función de los contextos socio-políticos. El uso estratégico del género implica tácticas diferenciales según las características de cada cual.

En efecto, Bachelet y Onkelinx viven en ambientes sociales muy distintos caracterizados por repertorios divergentes y logros de género diferenciados. Ser una mujer no significa lo mismo en Bélgica y en Chile, y las expectativas de género no son similares. Aún más, las mujeres gozan de diferentes derechos en cada uno de estos países (Franceschet, 2005). Por ejemplo, el aborto es todavía ilegal en Chile mientras que fue despenalizado en Bélgica en el año 1990, y no hubo ley de divorcio en Chile sino hasta el año 2005. Los procesos de individuación que garantizan la libre disposición del cuerpo propio en cual reside el corazón de la ciudadanía femenina han sido conseguidos en Bélgica mientras que en Chile es todavía un tema altamente sensible que cuenta con el rechazo decidido de los sectores conservadores y de centro manteniéndose solamente como un proyecto para 
la izquierda. Estas situaciones contrastadas suponen que el uso del género como un recurso político en la política es notablemente diferente en el caso de Chile y el de Bélgica.

Como mostraremos, la manera en que Bachelet y Onkelinx han construido su propio relato de género y tratan de hacerlo políticamente está profundamente moldeado por sus sociedades. De manera central para nuestro argumento, en ambos casos la maternidad aparece como un elemento clave en las presentaciones de sí, articulándola de manera particularmente diferenciada. Bachelet se presenta como una mujer en política, una estrategia que incluye subrayar en su construcción simbólica y de estilo algunos atributos asociados con la figura maternal, lo que, no obstante, no se reducen al complejo marianista de la madre (Chaney, 1992 $)^{7}$. Onkelinx trata de presentarse con la imagen de una mujer moderna, entendiendo por ello, una mujer que todavía es femenina, que cuida a sus niños, pasa tiempo con su compañero o esposo y que es profesionalmente independiente. El rol maternal que es al mismo tiempo reclamado es visto como algo intrínsecamente diferente a la política. Bachelet trata de demostrar que ella puede ser una buena política siendo una mujer mientras que Onkelinx intenta mostrar que ella puede ser una buena madre y una buena esposa y mantenerse como una mujer real al mismo tiempo que ser una política de primera línea. La presencia de ataques sexistas a Bachelet constituye un factor clave para explicar las divergencias con el discurso de Onkelinx. De hecho, Bachelet tenía que responder en primer lugar a esas críticas mientras que Onkelinx fue libre de desarrollar otro discurso. En breve, Bachelet necesitaba probar que ella podía ser una política sin dañar la política mientras que Onkelinx, libre de estos cuestionamientos, quería demostrar que ella podía ser una política sin dańar a la familia.

\section{Michelle Bachelet: ser mujer no es impedimento para la politica}

Michelle Bachelet (Santiago de Chile, 1951) comparte el perfil de otras líderes políticas en Chile, especialmente aquellas que pertenecen a la centro-izquierda de la Concertación de Partidos por la Democracia. Proviene de una familia de clase media, fue a una escuela pública y logró altos niveles educativos, tal como sus padres lo hicieron (Hardy, 2005). Lo único que la aleja de este perfil es la falta de redes familiares en política.

7 El culto a la superioridad espiritual femenina - "marianismo"- es una representación de lo femenino basada en la imagen católica de la Virgen María. Retrata a las mujeres como moralmente superiores y caracterizadas por su voluntad de sacrifico y devoción a los otros. 
Aun cuando su padre tuvo una posición en el gobierno de Salvador Allende fue esencialmente un oficial militar con una función administrativa. Apresado después del golpe de Estado en 1973, murió un año después. Michelle Bachelet y su madre fueron arrestadas en 1975 y una vez liberadas partieron al exilio, el que duró 4 años. De regreso a Chile Bachelet terminó sus estudios de medicina y desarrolló su carrera primero en una ONG y desde 1990 en el sistema de salud pública. En el 2000 el presidente Lagos la nombró Ministra de Salud y dos años después se convirtió en la primera Ministra de Defensa de América Latina. Después de dos años renunció para dedicarse a la campańa para la presidencia la cual ganó en 2006. De 2010 a 2013 fue Directora Ejecutiva de ONU Mujer. Actualmente es nuevamente presidenta de Chile para el período 2014-2018.

Su llegada al puesto de Presidenta de la República no fue resultado de una amplia discusión (Stoffel, 2005) o sensibilización de la clase política acerca de la importancia de aumentar la participación política de las mujeres. Fue efecto más bien del encuentro entre situaciones de facto, un espíritu pragmático y el cálculo político electoral. Las cúpulas de la Concertación no habían considerado inicialmente a Bachelet como una posible candidata. Fue el favoritismo de la ciudadanía expresado en diversas encuestas de opinión, el que definió en última instancia su candidatura. Bachelet misma se produce discursivamente como resultado del apoyo ciudadano, lo que es interpretado, por ella, además, como la voluntad del electorado de votar por una mujer. Ciertamente, varios signos de la emergencia de esta voluntad habían estado presentes en el comportamiento electoral en el transcurso de la década del noventa (Araujo, 2001, 2004; Navia y Sandoval, 1998), y se revelaron ya en las estrategias en juego en el proceso eleccionario 19992000 (Araujo, 2005).

Este favoritismo y luego el alto grado de simpatía y apoyo que Bachelet generó puede ser leído en una triple perspectiva. Primero, como efecto de la entronización de un nuevo tipo de liderazgo político. En consonancia con lo que ocurre a nivel internacional, el apoyo se dirige a liderazgos más cercanos y carismáticos, más vinculados a la vida cotidiana y a la gestión de lo privado, más espontáneos, sencillos y humildes (Tironi, 2005: 187-191). Las mujeres, y Bachelet en particular, en su doble condición de actoras políticas relativamente nuevas y de estar en posición de encarnar los atributos antes señalados serían quienes se beneficiarían de estas tendencias. Desde una segunda perspectiva, el apoyo puede considerarse resultado de una toma de distancia de la ciudadanía con las élites políticas, y, particularmente, de la desconfianza y rechazo a los partidos políticos. Bachelet, quien se presenta 
como una outsider si bien no de la política, sí de las élites concertacionistas (Ríos, 2008), tiene ventajas evidentes para recoger los réditos de esta posición. Tercera perspectiva explicativa: la condición de género de la candidata. Esta condición, de manera excepcional para el caso de líderes en cargos de su nivel, es movilizada por Bachelet retóricamente en la construcción de sí, cuestión inusual entre los líderes de esa posición, y en su lectura de la realidad nacional. En consecuencia, ella es favorecida por la identificación con ella de un sector importante del electorado femenino, lo que fue una de las causas de su victoria (Morales, 2008). Recoge, de esta manera, los efectos de transformaciones representacionales de género que se habían ido desarrollando a lo largo de casi quince años. Al mismo tiempo, sintoniza con la percepción que tienen las mujeres acerca de la inconsistencia entre estas nuevas representaciones y horizontes y sus experiencias cotidianas en ámbitos tan diversos como la familia, la pareja o el trabajo (Guzmán y Mauro, 2004, Sharim, 2005, Valdés et al, 2005; Guzmán y Godoy, 2009).

Pero son exactamente las mismas dimensiones que aportaron positivamente a su elección y apoyo, las que fueron la fuente de las críticas que debió sortear para poder legitimarse en el poder, especialmente en la primera parte de su gobierno. Las fuertes críticas tanto explícitas como implícitas fueron frecuentes. El desfase entre las expresiones de transformaciones en las representaciones ciudadanas y las convicciones aún apegadas a una forma tradicional de entender la sociedad y la política de parte importante de la clase política fue sin duda un aspecto central que determinó el tipo de retórica y estrategias de Bachelet. La construcción de sí estuvo fuertemente influida por el hecho que debió producirse de cara a los ataques que cuestionaron su estilo de liderazgo y su falta de experiencia y conocimiento del mundo político, ambos fuertemente asociados a su condición de género. Tal como ella mencionó al final de su período presidencial:

"Aquí ha habido gente de la política, hombres, de un cierto peso, pero eso era sinónimo de poderoso. En cambio, una mujer es una gorda. Si a un presidente, un hombre, en un momento muy emocionante se le llenaban los ojos de lágrimas, era un hombre sensible; en cambio, una mujer era una histérica. Estoy contando lo que salía en la prensa, no fantasías mías" (El País, 1/11/2009).

De esta manera, en el caso de Bachelet, el estatus de género reveló fuertemente su naturaleza ambivalente. Fue siempre un asunto relevante en su carrera política pero ha sido movilizado de formas distintas por los medios, los ciudadanos y la clase política. Por ejemplo, cuando ella fue 
Ministra de Defensa los medios enfatizaron repetidamente que ella estaba a cargo de una oficina tradicionalmente masculina ${ }^{8}$. Las representaciones de los medios de este nuevo poder femenino ganó la simpatía de los ciudadanos. Esto último se basó en lo que fue leído como su estilo femenino, amigable y cercano, y su habilidad para desarrollar un rol masculino como jefa de las fuerzas militares. De este modo, el género fue un elemento especialmente importante de distinción y aceptación. Sin embargo, las críticas de la clase política basadas en género y la desconfianza emergieron continuamente, especialmente tan pronto como Bachelet se convirtió en una seria candidata para la presidencia de Chile. La presencia de estas críticas se sintieron fuertemente durante la primera mitad de su período presidencial e incluso en los meses finales de su administración aunque, ciertamente, menos explícita y frecuentemente. De este modo, desde el comienzo el género fue un elemento políticamente ambiguo. Fue simultáneamente la fuente de la simpatía y el apoyo y al mismo tiempo de críticas y desconfianza política. Su percepción de la doble naturaleza del género se expresa a través de su período de gobierno.

Por ejemplo ella declaró en 2006: "Gobernaré con polleras y pantalones. Pantalones para tomar todas las decisiones firmes que hay que tomar y polleras para hacer que junto con empujar a los que quieren surgir, también acojamos y protejamos más a los que tienen dificultades" (El Mercurio, 16/01/2006). De manera similar ella aspiró a ser recordada por su estilo de conducción el cual describió "firme pero cálido" (El Mercurio, 22/10/2006).

A diferencia de otras mujeres políticas, como la belga Laurette Onkelinx, Bachelet enfrentó la situación haciendo uso máximo del género como un asunto mayor y como un instrumento de afirmación. En un contexto de críticas sexistas usó el género como un recurso político. Durante la campańa presidencial y su período en este cargo desarrolló una imagen fuertemente anclada en representaciones de género, jugando un rol decisivo en su trayectoria política y la construcción de su imagen pública (Subercaseaux y Sierra, 2005; Insunza y Ortega, 2005). Ella ha significado sus características como femeninas y ha estado constantemente relacionando sus experiencias sociales con el hecho de ser mujer. De esta manera, y a diferencia de otras líderes, Bachelet hizo del género un componente retórico y argumentativo de su vida política e imagen política. Esta actitud fue consistente con su particular sensibilidad con las cuestiones planteadas

8 Otro tema que fue enfatizado en este tiempo: el hecho que Bachelet haya tenido que trabajar con la institución que fue responsable de la muerte de su padre. Esto ayudó a forjar la imagen de ella como un ícono de reconciliación. 
por los movimientos de mujeres, tal como lo ha demostrado en su carrera política. Por ejemplo, cuando ella fue Ministra de Salud apoyó programas e iniciativas de contracepción y como Ministra de Defensa abordó la situación de las mujeres en las Fuerzas Armadas.

Discursivamente, Bachelet intentó impulsar una lectura positiva de las características femeninas (sin cuestionar si eran esencialmente femeninas); buscó producir un nuevo significado de estas características mucho más consistentes con las nuevas formas de hacer política. Estos esfuerzos fueron acompañados por un reconocimiento explícito de la importancia del machismo y la defensa de las políticas feministas en Chile. Por ejemplo, ella argumentó en una entrevista durante su campaña presidencial:

"Las mujeres se sienten discriminadas porque no siempre son reconocidos sus talentos y capacidades y porque aún se les castiga socialmente, porque además de ser buenas profesionales, tienen que tener la casa impecable y los niños perfectos... Hay muy pocas en la última plantilla parlamentaria, pero también hay pocas grandes empresarias. E incluso muchas veces he visto a mujeres dando grandes ideas sin ser reconocidas. Pero luego un hombre toma la misma idea, la redondea, la adorna un poquito y todo el mundo la encuentra genial” (Revista Cosas, 30/09/2005).

Estratégicamente Bachelet desarrolló tres acercamientos al género como recurso. Primero, usó su condición femenina como un elemento de identificación para construir proximidad a los ciudadanos y generar apoyo popular. Segundo, enfatizó la agenda de género en su gabinete. Finalmente, movilizó elementos maternales para construir su imagen política. La primera estrategia, fue usar el hecho de ser mujer como un elemento de identificación con otras mujeres y como una manera de reclamar su diferencia de los políticos tradicionales. En este contexto, el machismo de la clase política fue activamente denunciado especialmente, pero no exclusivamente, cuando los ataques de la oposición aumentaron. De hecho, su distancia con las elites políticas (aliadas y opositoras) fue crecientemente interpretada en términos de género. Por ejemplo, apareció la tesis del "femicidio político" después de las críticas que siguieron al sistema de transporte público urbano Transantiago y las acusaciones de corrupción en su gobierno que aparecieron el mismo año. Esta expresión, que fue usada por un periodista de izquierda (Cabieses, 2007), intentó presentar estas críticas como un caso de violencia masculina. De este modo, los ataques fueron explicados por el hecho de que ella era una mujer. Ese fue el más radical y explícito uso del género como explicación del 
trato recibido por buena parte de la clase política que Bachelet hizo durante en su carrera. Cuando esta estrategia falló, ella retrocedió y nunca volvió a intentar usar el género de manera tan radical.

La segunda estrategia fue el énfasis en la agenda de género de su gobierno (Valdés, 2010). Ésta la utilizó al menos dos maneras. De un lado, Bachelet le dio gran importancia a las cuestiones de género y comunicó en variadas formas y a través de canales distintos sus esfuerzos y logros en este dominio. Bachelet publicitó particularmente los logros de género en las situaciones de crisis, transformándolos en un instrumento para ganar su popularidad. De otro lado, y a diferencia de los tres presidentes anteriores, todos ellos de la Concertación, Bachelet adoptó una clara posición en términos de género, aun cuando pudieran ser controversiales. Por ejemplo, como ya lo dijimos, dio un aporte que era sin precedentes políticamente a los métodos de contracepción, un foco de gran debate en Chile (Las Últimas Noticias, 13/01/2007; El Mercurio, 31/10/2007).

La tercera estrategia fue empleada más fuertemente en la última parte de su período. Implicó fortalecer sus características de liderazgo y su estilo de manejo asociándolo directamente a la figura de la madre. Esta estrategia estuvo relacionada con los esfuerzos de alumbrar positivamente los aspectos de la feminidad. Mediante este discurso, conectó su trabajo a las características frecuentemente construidas como maternales, como son la cercanía, empatía, preocupación por los más débiles, calma y fuerza frente a las circunstancias adversas, una característica crucial en una región donde las figuras de la madre soltera y el padre ausente han sido ampliamente divulgadas (Montecinos, 1991).

Ciertamente, los rasgos maternales asociados a su estilo fueron puestos en juego en las representaciones de la prensa desde el inicio de su mandato. Mientras la prensa popular no dudó en llamarla "mamá" directamente (Las Últimas Noticias, 18/07/2006), otros diarios dirigidos a las élites hicieron uso de representaciones gráficas o asociaciones más sutiles que vinculaban su figura y sus propuestas de gobierno a la figura de madre por vía de los significantes protección, o la asociación de lo maternal con la preeminencia dada por la presidenta al fortalecimiento de políticas sociales (El Mercurio, 19/03/2006). Sin embargo, el uso de este tipo de rasgos para la construcción política fue retomada por la propia Bachelet más tarde. Sin embargo, es necesario subrayar que el uso retórico y estilístico de "madre" no implicó 
la restauración de los dotes tradicionales maternos, en tanto dimensión sacrificial o de sumisión -los cuales son centrales en el modelo mariano de madre que se ha indicado como presente en la política latinoamericana (Chaney, 1992; Montecinos, 1991). La figura de la madre es movilizada bajo nuevas coordenadas: fortaleza, calidez, cercanía y protección, pero no victimización y sacrificio.

Por medio de esta estrategia se colocó simbólicamente, y en consonancia con los rasgos asociados a la madre, por encima de los conflictos y las luchas políticas partidarias (sancionadas por la actitud presidencial como asuntos menores). Los rasgos maternales hicieron eco y recibieron consistencia en su relación con un discurso y una agenda en la que se privilegió la protección social (Valdés, 2010). En este contexto es importante hacer una distinción. Si bien Bachelet moviliza la asociación con la figura materna en la construcción de la imagen pública, no lo hace introduciendo su rol maternal efectivo. La producción de su imagen hace uso solamente de la asociación simbólica con los rasgos maternales. El rol maternal concreto, aun cuando es reivindicado, se presenta como algo intrínsecamente diferente a su función política. Lo anterior expresa otra diferencia importante, como veremos, con el caso de Onkelinx.

Bachelet es jefa de hogar y madre de tres hijos de dos padres distintos a los que ha criado sola con la ayuda de su propia madre, una realidad extendida en la sociedad chilena. Bachelet ha retomado con frecuencia la relación entre su maternidad y su puesto de presidenta a partir de la perspectiva de la deuda. La deuda adquirida con sus hijos, y nieto, a causa de sus obligaciones, es según ella, enorme, pero deberá esperar hasta el fin de su mandato para ser "reembolsada ". La familia es, así, colocada, aun cuando de manera conflictual, legítimamente en segundo lugar respecto de las obligaciones que ha contraído con el país. Como expresó en una entrevista en el ańo 2007:

"La verdad es que la posibilidad de estar a plenitud con los hijos está bastante limitada. Buscamos espacios, como cualquier familia. Pero uno sabe que estos años van a tener algunas insuficiencias y eso lo hemos conversado con los nińos. Alguien me dijo una vez que las mujeres podemos hacer todo, pero no todo al mismo tiempo. Antes, sentía que tenía que ser una súper woman y me frustraba cuando no podía ser una excelente dueńa de casa, una excelente mamá, una excelente profesional. Hacer todo eso al mismo tiempo, no es factible. Cuando uno aprende eso, vive más equilibradamente" (Revista Cosas, 10/2007). 
Una auténtica posición transgresiva respecto a las construcciones de género tradicionales aún vigentes en el país, las que colocan las funciones de cuidado familiar como exigencia y auto-exigencia normativa principal para las mujeres (Valdés, Castelain y Palacios, 2006).

Todas estas estrategias convergen en un aspecto central de la construcción de la imagen política de Bachelet: ella ha continuamente enfatizado su condición femenina mientras, significativamente, ha intentando mostrar simultáneamente que estas características asociadas con la feminidad no amenazan su trabajo político. De esta manera, y a diferencia de Onkelinx, como se verá, la estrategia principal de Bachelet ha sido reivindicar el género y constituirlo en un instrumento político. Pero, y esto es central, la importancia del género y sus atributos han sido movilizados en el cuadro de una estrategia retórica de afirmación constante que insiste en el hecho que ser una mujer no daña la política. Es precisamente en este sentido que se entiende, la otra gran diferencia con Onkelinx, el gesto retórico de producirse políticamente a través de signos maternales al mismo tiempo que la afirmación constante que su vida privada y sus labores maternales están en segundo lugar con respecto a las obligaciones que le impone su compromiso público.

\section{Laurette Onkelinx: ser una politica no daña a la familia}

Laurette Onkelinx (Ougrée, 1958) empezó su carrera política a mediados de los ochenta cuando fue elegida por primera vez en la Cámara de Representantes. Es hija de un conocido político socialista MP y sindicalista de Liège, Gastón Onkelinx, y fue presentada como tal al entrar a la política. Sin embargo, perdió rápidamente esta imagen y progresivamente ganó autonomía profesional. En 1992 se convirtió en Ministra de Integración Social, Salud Pública y Ambiente. Dos años después fue nombrada Ministra Presidente de la Comunidad Francesa de Bélgica a cargo del Servicio Público, Infancia y Salud. De alguna manera, fue considerado un castigo forzarla a dejar la política nacional. Sin embargo, ella mejoró la imagen de la institución de la Comunidad Francesa y fue confirmada en su puesto con mayores responsabilidades después de las elecciones regionales de 1995. Su fuerte oposición a los profesores secundarios que habían estado en huelga por varios meses le dio mayor relevancia política y le ayudó a desarrollar la imagen de una política senior. En 1999 se convirtió en Vice Primera Ministra del gobierno federal y en jefa de los socialistas francófonos, un cargo que todavía mantiene hasta hoy. Además tuvo bajo su responsabilidad 
las áreas de Empleo e Igualdad de Oportunidades desde 1999 hasta 2003, de Justicia desde 2003 hasta 2007, y de Asuntos Sociales y Salud Pública desde 2007. En 2009, ganó por segunda vez el título de la mujer más poderosa en Flandes del año, dada por la revista de negocios Trends (Le Soir, 6/11/2009). Dada la división del paisaje político belga a través de líneas culturales y lingüísticas (Deschouwer, 2009), el hecho que a una política francófona le fuera entregado tal premio puede ser interpretado como una demostración de su poder.

Onkelinx nunca se ha beneficiado ni por cuotas de género dentro de su partido ni por ley de cuotas o paridad. Sin embargo, ella ha desarrollado toda su carrera política en el contexto de un profundo cambio del lugar de las mujeres en la política en Bélgica (Marques-Pereira \& Gigante, 2001; Praud \& Henriques, 2008). Este ambiente legitimó, sin duda, la idea de un liderazgo femenino político y favoreció su acceso a los cargos más importantes del Estado belga. El debate de la participación política de las mujeres empezó durante este período. En este contexto Onkelinx introdujo las leyes de paridad de 2002 (Meier, 2005). Parcialmente, como resultado, la proporción de mujeres electas aumentó significativamente durante los años 2000 .

Durante su carrera política Onkelinx casi no ha sido víctima de ataques sexistas. Los periódicos han producido muy pocas representaciones generizadas negativas respecto a ella y su trabajo. Los reportes sobre sus acciones tienden a ser neutrales, al menos en lo que concierne al género. $\mathrm{Al}$ comienzo de su carrera ella fue presentada como bonita y elegante y los columnistas usaban con frecuencia la expresión "joli minois" (pequeña y bonita cara). Los periodistas informaban a los lectores acerca de su sonrisa, su cabello, sus ojos, su juventud, etc. Después de un tiempo ella fue crecientemente presentada como la dama de hierro con una alegre sonrisa, una imagen aún hegemónica en los medios belgas hoy. Por ejemplo, una periodista la describió de esta manera en 2002: "su pequeña cabeza rebelde se mueve mucho. Sus labios pintados de rojo permiten filtrar los flujos de palabras. Madame, la diputada primer ministro se mueve sin hacer ruido, firmemente" (Vers L'Avenir, 13/06/2002).

La ausencia de críticas sexistas implica, que a diferencia de la situación de Bachelet, Onkelinx no estaba obligada a hablar de género, ni a producir una defensa de sí en estos términos. El género no era un asunto central y ella podría probablemente haber desarrollado toda su carrera política sin haberse referido a él. Sin embargo, no fue el caso. Esto se grafica por un lado, por 
la combinación entre la atención que en los medios se le dio a su apariencia, y al hecho que ella la cambia regularmente. Onkelinx no solo cambia su apariencia sino que, de manera más importante, ella quiere ser percibida como femenina. Desde aquí ella aparece como un contraejemplo respecto de la versión crítica de las mujeres políticas como masculinizadas. Insiste en la dimensión física de la feminidad al alterar su corte de pelo o a través del cuidado con que ella elige su ropa. Simultáneamente, sostiene públicamente que las mujeres se comportan diferentemente y, de esta manera, podrían traer algo diferente a la política (Le Soir, 27/04/1992).

De este modo, los intereses por subrayar su condición de género no se han limitado a la apariencia. Ella ha comunicado sobre género principalmente haciendo un puente entre su vida y su puesto político. Es decir, poniendo el acento en la conciliación entre el mundo del trabajo y la familia. Este acento, más que el resultado de una convicción programática asociada al feminismo, es presentado como un espejo de sus creencias íntimas. Es un problema personal, por medio del cual se procura la identificación de otras mujeres de su sociedad, que es colocado como un tópico de su agenda política. Onkelinx nunca perteneció al movimiento feminista y no mostró especial interés por estar a cargo del departamento de Igualdad de Oportunidades. Raramente ha empujado asuntos vinculados al movimiento feminista a menos que le haya sido solicitado por su partido, a diferencia de otras políticas belgas como Miet Smet (Cockx, 2009). Por ejemplo, ella apoyó la introducción de la paridad de género en la constitución belga de 2002, pero ésta fue una parte del manifiesto de su partido. Más allá de ello, Onkelinx nunca apareció realmente interesada por el debate de la participación política de las mujeres, y no tocó este tema con los periodistas a menos que ellos lo pusieran. Adicionalmente, en estos casos, ella ha respondido convencionalmente reflejando el punto de vista de su partido en el tema. Además, tiende a descartar el carácter crucial de esta desigualdad en política considerándola un legado del pasado. Algunos años atrás, por ejemplo, ella declaró:

“...al comienzo cuando fui nombrada a un ministerio recuerdo que un oponente político me dijo "no es un portafolios, es una cartera". Mis amigas en Francia, Martine Aubry y Elisabeth Guigou, me dijeron que, en el Parlamento francés, hay giros increíbles y un montón de machismo en las palabras. Aquí eso no es el caso más" (Victoire/Le Soir, 4/10/2003). 
De manera evidente, las críticas generizadas de la clase política están lejos de constituir el contexto de su acción, como sucede con la figura de Bachelet. Más aún, en este caso, se encuentra incluso la imagen del mundo de la política belga como un espacio libre de sexismo y machismo.

Su sensibilidad por aspectos que tocan a las mujeres es más práctico: está anclado en la vida cotidiana. No en el mundo del trabajo sino en la interface entre el trabajo y la casa (Humo, 30/09/2003). En consonancia, a través de su carrera ha privilegiado cuestiones de género que tienen que ver con la maternidad y la familia. En efecto, desde sus inicios en política, ella ha insistido en la dificultad de las mujeres para articular vida profesional y vida familiar, y la conciliación entre trabajo y familia aparece como un leimotiv. El interés personal de esta temática se revela en que aun cuando no fuera preguntada por estas cuestiones o por su vida como política mujer, ella encontró la manera para evocar este asunto confirmando que ella lo consideraba una prioridad. En 1992, bastante temprano en su carrera, ella explicaba ya al periódico Le Soir que:

"Es verdaderamente difícil conciliar el trabajo y la vida familiar. Incluso con un esposo extremadamente presente. Yo no quisiera tener ningún otro mandato para poder dedicar la mayor parte de mi tiempo y mi familia a mis amigos y a mis "social permanences" para permanecer en contacto con la realidad” (Le Soir, 27/04/ 1992).

Ella ha repetido este mensaje a través de su carrera como lo muestra una entrevista más reciente:

"Yo no sumo el puesto de ministro con otro mandato. Es una elección. Todo mi tiempo libre está dedicado a mi familia. Soy extremadamente organizada. Mi esposo me ayuda mucho. Él trae sus dossiers a la casa y está muy presente. Como alguien me ayuda con el trabajo doméstico y como mi esposo siempre cocina, cuando vuelvo a la casa puedo dedicarme enteramente a los nińos. Estoy aprendiendo latín de nuevo para ayudar a mi hijo" (La Capitale, 10-11/05/2003).

Este énfasis en la dificultad para combinar trabajo y vida en familia revela la importancia que la maternidad y la familia ocupa en la vida de Onkelinx y su mensaje político. Este reto es al mismo tiempo presentado como una experiencia personal -es una de las pocas cosas que

9 Corresponden a las horas en las que los ciudadanos y las ciudadanas pueden ir a encontrarse directamente con los políticos y políticas. Generalmente se desarrollan en los cafés. 
ha declarado sobre su vida privada- y como una cuestión colectiva que todas las mujeres activas están enfrentando y que requiere de una voluntad política para ser modificada. La familia y la maternidad están en el centro de sus preocupaciones y sus discursos, no obstante, la manera en que ella habla sobre maternidad y como la usa políticamente difiere del discurso y estrategia de Bachelet. De hecho, aun cuando apela al rol de madre y concibe la maternidad como un asunto crucial en la vida de las mujeres, ella no moviliza los "dones maternales" en su acción política. Ella presenta sus roles en la familia y en la política como fundamentales pero distintos. Tal como ella lo menciona:

“...esta no es una confesión nueva pero yo realmente estoy obsesionada por el reto de manejar tres roles que son igualmente importantes para mí: aquel de ministra, mujer y madre. Requiere mucho tiempo de gestión. Cuando yo consigo tener éxito con eso entonces alcanzo la armonía porque estar completamente realizada como mujer y como madre amorosa me ayuda en política y viceversa" (L’Écho, 14/09/ 2002).

Retrata a la familia como un reducto de paz y un elemento clave para su bienestar, "un lugar para relajarse, un lugar para amar, un lugar que me permite realizar mis viajes internos más bellos" (Vers L'Avenir, 19/09/2002). Estas declaraciones ponen en claro que ella considera a la familia como totalmente extranjera al trabajo y que puede ser de alguna manera salvaguardada. Se podría señalar que ella está redefiniendo la división entre la política y la familia, lo que puede contribuir a la reprivatización de la última.

Las declaraciones sobre su vida sentimental ${ }^{10}$, aun cuando escasas, hacen eco de aquellas sobre la familia. Por mucho tiempo evitó mencionarla públicamente, pero su relación con un prominente abogado socialista, quien trabaja regularmente para este partido, la que fue seguida por una ampliamente comentada boda, la obligó a tratar el asunto. Nuevamente, ella presentó su vida sentimental como algo intrínsecamente diferente a su trabajo aun cuando de manera evidente hay un caso de superposición. Onkelinx presenta su relación actual en el marco de un relato de amor romántico. Su versión es que se divorció porque ya no estaba más enamorada de su esposo anterior. Marc, su pareja actual, y ella se conocieron a través

10 Onkelinx se ha casado dos veces. Su primer matrimonio con Abbes Guenned terminó en divorcio en 1997, contrayendo segundas nupcias con Marc Uyttendaele en 1998. 
de amigos comunes y se enamoraron a primera vista. Su relación es descrita como armoniosa, regida por el amor y el respeto. El relato insiste en separar la historia de amor del trabajo y con ello descartar las críticas que han tildado su boda como una relación de negocios y que han puesto sobre la mesa los riesgos potenciales de colusión, especialmente porque en aquel momento ella era ministra de justicia (Télémoustique, 17/11/2004).

A través de estas declaraciones, Onkelinx busca dar una imagen de mujer moderna. Esto es, profesionalmente activa que simultáneamente se mantiene femenina, una buena madre y una compañera cuidadosa. Reclama ser una “mujer realizada” y "gozadora” (Het Laatste Nieuws, 27/11/2004). Esto es confirmado por la manera en que describe la admiración que le suscita una de sus colegas de gobierno, la joven socialista flamenca Freya Van den Bosche. Ella la encuentra fascinante porque ha conseguido tener éxito en un desafío muy difícil:

“...ser terriblemente mujer y sé decir abiertamente que ella coloca su vida de familia en un pedestal. Ella nunca olvida proteger a sus hijos... Ella es una mujer de convicción, inteligente activa y trabajadora. No tiene miedo de romper tabús. Naturalmente, dice lo que piensa y no habla sandeces políticas. Todo eso junto hace de ella un fenómeno: alguien a quien a una le gustaría parecerse" (Victoire/ Le Soir, 4/10/2003).

El discurso de Onkelinx podría ser descrito como un esfuerzo por demostrar que ella puede ser una buena madre (y una buena compañera) al mismo tiempo que está profesionalmente activa. Lo central en ella es la declaración que su familia no es afectada por su carrera política. Al hacerlo, al mismo tiempo, retóricamente apela a la identificación y cercanía con las otras mujeres. Como lo planteó en 2003: "yo he tratado de hacer claro que soy una mujer como otras. Si, yo estoy enamorada, yo tengo niños, tengo problemas en mi vida, etc." (Victoire/Le Soir, 4/10/2003).

Las imágenes que Onkenlinx ha intentado transmitir, la de una mujer activa que tiene tiempo para sus hijos y su pareja o esposo, así como su preocupación de conciliar el trabajo y la vida familiar; son las que probablemente reflejan sus creencias internas. Al mismo tiempo, sistemáticamente lo ha puesto en un contexto en el que no estaba obligada a referirse al género. Por ende, el género no aparece como un obstáculo a la brillante carrera política de Onkenlinx, sino como un recurso político, que ha sido movilizado entre otros medios políticos. De otro modo, 
probablemente no se hubiera referido tan sistemáticamente al tema de la conciliación entre trabajo y familia, incluso si pensaba que era lo correcto de hacer. Esta investigación también demuestra que ella se ha referido a este asunto desde su experiencia privada, conectándola a la vida diaria de muchas otras mujeres belgas. A través de esta estrategia, ha construido una cierta imagen de mujer, formada en una específica cuenta de género. Al mismo tiempo, estas creencias están enraizadas en el discurso del Partido Socialista sobre la mujer, así como en los principales repertorios de género de la sociedad belga. Este entrelazamiento es decisivo para aquellos discursos que resuenan con las creencias de los militantes socialistas al mismo tiempo que en el interior de la sociedad belga. Onkelinx se presenta como una mujer exitosa y plenamente activa en el mundo público que no descuida su familia, ni como madre ni como mujer.

\section{Conclusiones}

A través del caso de Michelle Bachelet y Laurette Onkelinx hemos tratado de acercarnos a las formas en que el género es movilizado en la presentación de las mujeres en los cuerpos ejecutivos. Sin embargo, lejos de lo que hace la mayor parte de la literatura hemos decidido no enfocarnos en las maneras que estas representaciones expresan valores patriarcales y evitan que las mujeres lleguen a los altos cargos políticos así como obstaculizan el desarrollo de las carreras políticas. A pesar de que no se trata de negar la existencia de barreras sistémicas al acceso de las mujeres a los cargos políticos, el artículo subraya en el contexto de las transformaciones en este ámbito de qué manera el género puede ser concebido como un recurso en la construcción política de la imagen de sí al mismo tiempo que las diferentes modalidades en que éste puede ser movilizado. En este artículo hemos puesto énfasis en la manera en la cual prominentes mujeres políticas han hecho más bien un uso estratégico de su condición de mujeres para impulsar sus carreras políticas. Este acercamiento implica moverse de la visión de mujeres como víctimas a una que subraye su agencia.

Nuestro análisis muestra que más allá del uso común del género como recurso político, Bachelet y Onkelinx lo usaron de manera diferente. La primera invoca los atributos de género para demostrar que puede ser una buena política que no va a dañar la política porque sus cualidades femeninas contribuyen a enriquecerla. La última reclama que su vida política no afecta su vida familiar, porque ella es capaz de preservar tiempo para su compañero/esposo y sus niños. Más allá de esto, ella intenta expandir la 
imagen de una mujer femenina que combate el estereotipo de una política femenina masculinizada. Como ha sido sugerido aquí, estas diferencias en el uso estratégico de género son reflejo de los distintos debates nacionales sobre género y política, así como los repertorios de género y los logros de género distintos en cada sociedad. Los ataques sexistas enfrentados por Bachelet han contribuido a explicar el contenido de esta estrategia.

Finalmente, debe ser subrayado que en los dos casos la maternidad es central en la definición de género, que ambas, Bachelet y Onkelinx, lo han movilizado en su esfuerzo de tomar ventaja de su condición de mujeres. La actual presidenta chilena ha invocado las virtudes maternales en su trabajo político mientras que la vice primer ministra belga ha presentado a su familia como un oasis en donde ella puede tener una pausa para volver a la política con nuevas ideas y mayor frescura. Según ella, la maternidad es claramente distinta de la política pero ambas son indispensables para su bienestar personal. Una centralidad de la maternidad que nos retrotrae a los debates feministas clásicos. Los casos estudiados muestran la persistencia de la maternidad y la figura de la madre como elementos de la configuración de las imágenes de estas mujeres políticas, pero también revelan que su peso y su cualidad en las formas de producción de sí y como recurso han variado significativamente. Lo maternal parece como instrumento privilegiado (y exitoso) de feminización de la esfera política, pero, también, la maternidad se revela como elemento constitutivo de la preocupación por formas de producción equilibradas de sí. En ambos casos, un elemento principal del género como recurso político.

Si la maternidad se muestra en estos casos, siendo un elemento crucial en la producción de la imagen generizada de las mujeres en política, se abre la interrogante sobre cómo deben ser leídos los eventuales efectos de este hecho. ¿Debemos concebirlos aún en clave puramente de subordinación, o, más bien, debemos re-considerar la presencia de estas dimensiones maternales en el contexto de las transformaciones estructurales y culturales acontecidas en las últimas décadas leyéndolas fuera de esta clave? Un trabajo como el desarrollado impide una respuesta definitiva sobre este punto, pero deja abierta la pregunta. 


\section{Referencias bibliográficas}

Araujo Kathya (2004), "Mujeres y representación política: el caso de Chile", en Kathya Araujo, Nélida Archenti, Roció Villanueva y Bérengère MarquesPereira, Igualdad de Derechos, Igualdad de Oportunidades, Igualdad de Ejercicio, Santiago, Fundación Instituto de la Mujer, p. 101-127.

(2005), "Significations et representations imaginaires relatives aux femmes dans l'espace politique chilien (1999-2003)", Cabiers du GELAIS, No 5, p.159-186.

Archenti Nélida y María Inés Tula, editoras (2008), Mujeres y política en América Latina. Sistemas de cuotas de género, Helista, Buenos Aires.

Bauer Gretchen \& Manon Tremblay (2011), Women in Executive Power:A Global Overview, Routledge, Londres.

Bonnafous Simonne (2003), 'Femme politique'. Une question de genre? Réseaux, No 20, p. 119-145.

Borelli MaryAnne (2002), The President's Cabinet. Gender, Power and Representation, Lynn Riener, Boulder.

Cabieses Manuel (2007), "Femicidio político", Revista Punto Final, No. 645, Disponible en http://www.puntofinal.cl/645/femicidio.htm (visitada 25/08/2010).

Celis, Karen Mona Lena Krook \& Petra Meier (2011), “The Rise of Gender Quotas Laws: Expanding the Spectrum of Determinants for Electoral Reform", West European Politics, Vol.3, No. 34, p. 514-530.

Chaney Elsa (1992), Supermadre: la mujer dentro de la politica en América Latina, FCE, México.

Clift, Eleonor \& Tom Brazaitis (2003), Madam President : Women Blazing the Leadership Trail, Routledge, Nueva York.

Conway Daniel (2011), "Margaret Thatcher, Dress and the Politics of Fashion", Paper presentado en London College of Fashion, Disponible en http://www.fashion.arts.ac.uk/media/research/documents/daniel-conwaybritish-politics.pdf (visitada 01/03/2012). 
Cockx Romy (2009), Miet Smet: Trois décennies de politique d'égalité des chances, Institut pour l'égalité des femmes et des hommes, Bruselas.

Coulomb-Gully Marlène, editora (2009a), "Présidentielle 2007. Scènes de genre", Mots. Les langages du politique, No 90.

(2009b), "Le corps présidentielle. Représentation et incarnation dans la campagne présidentielle française de 2007", Mots. Les langages du politique, No 89, p. 25-38.

Deschouwer Kris (2009), The Politics of Belgium: Governing A Divided Society, Palgrave McMillan, Basingstoke.

Doran Marie-Christine (2010), "Les femmes et la politique au Chili: La dynamique et l'impact de l'accession au pouvoir de Michelle Bachelet", Recherches feminists, Vol. 23, No.1, p. 9-27.

Dulong Delphine \& Sandrine Lévêque (2002), "Une resource contingente. Les conditions de reconversion du genre en resource politique", Politix, Vol. 60, No.1, p.61-111.

Escobar-Lemmon María \& Michelle Taylor-Robinson (2009), "Getting to the Top. Career Paths of Women in Latin American Cabinets”, Political Quartely, Vol. 62, No.4, p.685-699.

Escobar-Lemmon María \& Michelle Taylor-Robinson (2005), "Women Ministers in Latin American Government: When, Where and Why", American Journal of Political Science, Vol. 49, No.4, p.829-844.

Franceschet Suzan (2010), "Continuity or Change? Gender Policy during the Bachelet Administration”, en Silvia Borzutzky \& Gregory Weeks, editores, The Bachelet Government: Conflict and Consensus in Post-Pinochet Chile, University of Florida Press, Gainesville, p. 159-180.

(2006), "El Triunfo de Bachelet y el Ascenso Político de las Mujeres”, Nueva Sociedad, No. 202, p. 13-22.

(2005), Women and Politics in Chile, Lynne Riener,

Boulder.

Franceschet Suzan \& Gwynn Thomas (2010), "Renegotiating Political Leadership: Michelle Bachelet's Rise to the Chilean Presidency", en Rainbow 
Freedman Jane (1997), Fermmes politiques : Mythes et symboles, L'Harmattan, París.

Guzmán Virginia y Amalia Mauro (2004), “Las trayectorias laborales de mujeres de tres generaciones: coacción y autonomía", en Rosalba Todaro y Sonia Yáñez, editoras, El trabajo se transforma. Relaciones de producción y relaciones de género, CEM, Santiago, p. 207-245.

Guzmán Virginia y Lorena Godoy, “Individuación y normatividad de género: la construcción de proyectos biográficos de mujeres”, en Kathya A raujo, editora, Se acata pero no se cumple. Estudios sobre las normas en América Latina, LOM, Santiago, p.175-197.

Hardy Clarisa (2005), Liderazgos femeninos en Chile, Catalonia, Santiago.

Insunza Andrea y Javier Ortega (2005), Bachelet. La historia no official, Random House Mondadori/UDP, Santiago.

Huddy Leonie \& Nayda Terkildsen (1993), "Gender Stereotypes and the Perception of Male and Female Candidates", American Journal of Political Science, Vol. 37, No.1, p. 119-147.

Jalalzai Farida (2008), "Women Rule: Shattering the Executive Glass Ceiling”, Politics and Gender, Vol. 4, No.2, p. 205 - 231.

Jalalzai Farida \& Mona Lena Krook (2010), "Beyond Hillary and Benazir: Women's Political Leadership Worldwide”, International Political Science Review, Vol. 31, No.1, p. 5-23.

Krook Mona Lena, (2010), Quotas for Women in Politics. Gender and Candidate Selection. Reform Worldwide, Oxford University Press, Oxford.

Llanos Beatriz y Kristen Sample, (2008), 30 años de democracia: ¿en la cresta de la ola? Participación politica de las mujeres en América Latina, IDEA, Estocolmo.

Lovenduski Joni (2005), State Feminism and Political Representation, Cambridge University Press, Cambridge. 
Marques-Pereira Bérengère (2011), “De la féminisation des démocraties au genre comme ressource politique. Le cas chilien", Cabiers du Genre (horssérie), p. 115-134.

(2007a), "Les femmes latino-américaines dans les exécutifs nationaux. Le cas du Chili”, Histoire@Politique, Politique, Culture, Société, Revue électronique du Centre d'histoire de Sciences Po, No. 1, Disponible en http://www.histoire-politique.fr/index.php?numero=01\&rub =dossier\&item $=8$ (Visitada 21709/2011).

(2007b), "La citoyenneté politique des femmes dans un jeu de miroir entre l'Europe et l'A mérique latine”, en Manon Tremblay, Thanh-Huyen Ballmer-Cao, Bérengère Marques-Pereira \& Mariette Sineau, editoras, Genre, citoyenneté et representation, Les Presses de l'Université Laval, Sainte-Foy, p. 23-40.

(2003), La citoyenneté politique des femmes,

Armand Collin, París.

Marques-Pereira Bérengère \& Catherine Gigante (2001), "La représentation politique des femmes : des quotas à la parité ?", Courrier hebdomadaire du CRISP 1723.

Marques-Pereira Bérengère \& Patricio Nolasco, editores (2001), La représentation politique des femmes en Amérique latine, L'Harmattan, París.

Mateo Diaz Mercedes (2005), Representing Women? Female Legislators in West European Parliaments, ECPR Press, Colchester.

Meier Petra (2005), “The Belgian Paradox: Inclusion and Exclusion of Gender Issues”, en Joni Lovenduski, Claudie Baudino, María Guadaguigni, Petra Meier \& Diane Sainsbury, editores, State Feminism and Political Representation, Cambridge University Press, Cambridge, p. 41 - 61.

Montecinos Sonia (1991), Madres y Huachos, Cuarto Propio/Cedem, Santiago.

Morales Mauricio (2008), “La primera mujer presidenta de Chile: ¿Qué explicó el triunfo de Michelle Bachelet en las elecciones de 2005-2006?", Latin American Research Review, Vol. 43, No.1, p. 7 - 32.

Murray Rainbow, editora (2010), Cracking the Highest Glass Ceiling: Women's 
Campaigns for Executive Office Worldwide, Praeger, Santa Bárbara.

Navia Patricio y José Miguel Sandoval (1998), "Las mujeres y las elecciones parlamentarias 1993 en Chile”, en María Luisa Tarrés, editora, Género y Cultura en América Latina, Colegio de México, México, p. 243-260.

Pateman Carol (1988), The Sexual Contract, Stanford University Press, Stanford.

Philips Anne (1995), The Politics of Presence, Clarendon Press, Oxford. Praud Jocelyne \& Karl Henriques (2008), "Constitutionalizing and Legislating Parity Democracy: The Cases of France and Belgium", Public Policy Paper (Saskatchewan Institute of Public Policy), No. 56.

Ríos Marcela (2008), "Seizing a window opportunity. The election of President Bachelet in Chile", Gender and politics, Vol. 44, No. 3, p. 496-509.

Sharim Dariela (2005), "La identidad de género en los tiempos de cambio: una aproximación desde los relatos de vida", Psykhe, Vol. 14, No. 2, p. 19-32.

Sineau Mariette (2011), Femmes et pouvoir sous la Ve République: De l'exclusion à l'entrée dans la course présidentielle, Presses de Sciences Po, París.

Stoffel Sophie (2005), "Le débat chilien sur la représentation politique des femmes et sur le projet d'une 'loi des quotas", Cahiers du GELA-IS, No. 5, p. $21-49$.

Subercaseaux Elizabeth y Malu Sierra (2005), Michelle, Catalonia, Santiago. Thomas, Gwynn (2011), "Michelle Bachelet's Liderazgo Feminino (Female Leadership)". International Feminist Journal of Politics, Vol.13, No.1, p. 63 82.

Thomas Gwynn \& Melinda Adams (2010), "Breaking the Final Glass Ceiling: The Influence of Gender in the Election of Ellen Johnson-Sirleaf and Michelle Bachelet", Journal of Women, Politics and Policy, Vol.31, No.2, p. 105-31.

Tironi Eugenio (2005), Comunidad, familia y nación en el Bicentenario. El sueño chileno, Taurus, Santiago. 
Tremblay, Manon, editora (2008), Women and Legislative Representation, Palgrave MacMillan, Nueva York.

Valdés, Ximena et al. (2005), "Entre la reinvención y la tradición selectiva: familia, conyualidad, parentalidad y sujeto en Santiago de Chile”, en Teresa Valdés y Ximena Valdés, editoras, Familia y vida privada. ¿Transformaciones, tensiones, resistencias y nuevos sentidos?, FLACSO-Chile/Cedem/UNFPA, Santiago, p. 163-213.

Valdés, Ximena, Christine Castelain-Meuniers y Margarita Palacios (2006), Puertas adentro. Masculino y femenino en la familia contemporánea, LOM, Santiago.

Valdés, Teresa, editora (2010), ¿Género en el Poder? El Chile de Michelle Bachelet, LOM, Santiago.

Valenzuela, Sebastian \& Teresa Correa (2009), "Press coverage and public opinion on women candidates: The case of Chile's Michelle Bachelet", The International Communication Gazette, Vol. 71, No.3, 203 - 223.

Vera Guajardo, Antonieta (2008), "Les discours du genre dans la campagne présidentielle de Michelle Bachelet : une critique féministe", Raisons politiques, Vol. 31, No.3, p. 81 - 103.

Young, Iris (2000), Inclusion and Democracy, Oxford University Press, Oxford.

\section{Periódicos y revistas}

1992. “Les passions de Laurette Onkelinx”. Le Soir. 27 de abril.

2002. "Laurette Onkelinx, la rose pourpre”. L'Echo. 14 de octubre.

2002. “Madame a du mordant et des yeux pétillants". Vers l'Avenir. 13 de junio.

2002. “Laurette Onkelinx: Ses plus beaux voyages intérieurs”. Vers l'avenir. 19 de septiembre.

2003. "Fête des mères : Comment font-elles ? Maman et politicienne: un exploit”. La Capitale. 10 - 11 de mayo. 
2003. "Rode oortjes: Humo sprak met Laurette Onkelinx (PS), Minister van Justitie: 'Thuis ben ik een heel andere vrouw: dan is er liefde in het spel'”. Humo. 30 de septiembre.

2004. “Laurette Onkelinx : "Une partie de la Belgique a la mémoire courte...”. Telemoustique. 17 de noviembre.

2004. "Ah, la Belgique. Hoe lang nog, vraag ik me soms af “. Het Laatste Nieuws. 27 de noviembre.

2005. "Michelle Bachelet: me siento una privilegiada". Revista Cosas. 30 de septiembre.

2006. “La primera mujer que gobernará Chile”. El Mercurio. 16 de enero.

2006. “Tacones cercanos”. El Mercurio. 22 de enero.

2006. "El turno del Estado maternal”. El Mercurio. 19 de marzo.

2006. "Bachelet: es deber del Estado entregar distintas opciones de anticoncepción". www.cooperativa.cl. 23 de marzo.

2006. “Mamá Bachelet se subió al tanque y paro en seco a "Larraín y Larraín”. Las Últimas Noticias. 18 de julio.

2007. “Michelle desafía a la Iglesia Católica. Firmará decreto Supremo para distribuir la píldora del día después”. Las Últimas Noticias. 13 de enero.

2007. "Las confesiones de Michelle Bachelet: hay una campaña de femicidio a la figura presidencial". Revista Cosas. Octubre.

2007. Bachelet responde críticas de la Iglesia por venta de la píldora del día después. El Mercurio. 31 de octubre.

2009. "Entrevista Michelle Bachelet. Presidenta de Chile: Los hombres sienten más una atracción fatal por el poder”. El Pais. 1 de noviembre.

2009. “Onkelinx toujours la plus puissante en Flandre”. Le Soir. 6 de noviembre.

Vanhoenacker, Charline.“« Elle attire la lumière » (entretien)”. Victoire/Le Soir. 4 de octubre 2003. 